\title{
Duty-cycle division multiplexing (DCDM): a novel and economical optical multiplexing and electrical demultiplexing technique for high speed fiber optics networks
}

\begin{abstract}
A new multiplexing and demultiplexing technique based on duty cycle division is proposed, thus the name duty cycle division multiplexing (DCDM). DCDM can be applied in both electrical and optical domains. The new technique allows for more efficient use of time slots as well as the spectrum, taking advantage of both the conventional TDM and FDM. In this paper, three channels operating at the same speed of 10 Gbps are multiplexed in the optical domain and demultiplexed in the electrical domain. The performance comparison is made against $30 \mathrm{Gbps}$ TDM, and the experimental simulation results show that the minimum sensitivity achieved is $-26 \mathrm{dBm}$ and $-25.5 \mathrm{dBm}$ for the two systems respectively, thus a 0.5 dB improvement.
\end{abstract}

Keyword: Duty cycle division; Duty cycle division multiplexing (DCDM); Optical communication; Demultiplexing; Multiplexing; Computer networks 